\title{
Knowledge of Nurses Regarding Essential Newborn Care in Special Care Neonatal Units (SCANU) of Different Hospitals of Chattogram, Bangladesh
}

\author{
T SHARMIN ${ }^{\mathrm{a}}$, P K CHOWDHURY ${ }^{\mathrm{b}}$, TH KHAN $^{\mathrm{c}}$, O HOQUE ${ }^{\mathrm{d}}$
}

\begin{abstract}
:
Background: The advancements in intensive care in recent decades have enabled better survival of newborn. Knowledge of essential newborn care is important for the survival, growth, and development of a newborn. In spite of its essentiality, most health-care professionals do not know and follow the World Health Organization recommendation.

Aim and objectives: The aim of this study was to assess the knowledge of nurses regarding essential newborn Care in Special Care Neonatal Units (SCANU).

Materials and methods: This observational study was conducted among the nurses working in SCANU of Chattogram Medical College and Hospital, Chattogram MaaO-Shishu Hospital and Bangabandu memorial hospital of University of Science and Technology, Chattogram (USTC) for 6 months. Purposive sampling technique were applied for selecting the sample. A structured data sheet was predesigned to collect data. Knowledge regarding Essential Newborn Care among the nurses graded as satisfactory or not satisfactory.

Results: In this study it was observed that $69.0 \%$ nurses had completed diploma in nursing and midwife education, $B$.
\end{abstract}

Introduction:

New born care is of immense importance for healthy life and proper development of a baby. Globally, 2.4 million

a. Dr. Tasnuva Sharmin, Registrar, Pediatrics, Dhaka Medical College and Hospital Dhaka.

b. Prof. Pranab Kumar Chowdhury, Ex Head of the Department of Pediatrics Chittagong Medical College and Hospital, Chittagong.

c. Dr. Tafazzal Hossain Khan, Associate Professor, Department of Neonatology Dhaka Medical College and Hospital, Dhaka.

d. Dr. Md. Obidul Hoque, Medical Officer, Sheikh Fazilatunnesa Mujib Eye Hospital \& Training Institute, Gopalganj.

Address of Correspondence: Dr. Tasnuva Sharmin, Registrar Pediatrics, DMCH. Flat - 20-A-7, Concord Twin Tower, Santinagar. Dhaka. Cell: 01716741839, E-mail: tasnuvasharmin1983@gmail.com

Received: 30 Sept., 2020

Accepted: 05 May, 2021
Sc in nursing 11.0\%, diploma in nursing 7.0\%. $56.0 \%$ nurses attended special courses/training in neonatology. $\mathbf{9 4 . 7 5 \%}$ of the nurses had satisfactory knowledge regarding essential newborn care. $58.0 \%$ of nurses washed their hands with disinfectant solution before handling the baby, 99.0\% mentioned that the baby must be breast feeding within $1^{\text {st }}$ hour of delivery, 92.0\% mentioned that baby must be fed 6 months of exclusive breast feed, $91.0 \%$ knew how to stabilize body temperature of low birth weight newborn. The knowledge regarding essential newborn care were almost similar with age, gender, religion, marital status, educational level, working place and attending special courses/training in neonatology.

Conclusion: Most of the nurses working in SCANU of both Government and non-government hospitals of Chattogram have good knowledge regarding ENC. This knowledge appeared irrespective of their age, gender, religion, marital status, working place and special courses/training in neonatology.

Key words: Knowledge, Essential newborn care, SCANU

(J Bangladesh Coll Phys Surg 2021; 39: 185-192) DOI: https://doi.org/10.3329/jbcps.v39i3.54163

children died in the first month of life in 2019 approximately 6,700 neonatal death everyday -with about a third of all neonatal death occurring within the first day after birth and close to three-quarters occurring within the first week of life. ${ }^{1}$ Ninety percent of these deaths are take place in developing countries. ${ }^{2}$ The neonatal mortality rate is 30 deaths per 1,000 live births; it accounts for $67 \%$ of all under- 5 deaths. ${ }^{3}$ Infections, birth asphyxia, prematurity and low birth wt are major causes of death within this period. ${ }^{4} \mathrm{New}$ born complications resulting from hypothermia, infection and birth asphyxia that occur within the first seven days, following birth contribute to the highest burden of morbidity and mortality. ${ }^{5}$

Essential newborn care is a set of recommendations out lined by World Health Organization (WHO)which includes thermoregulation, clean delivery, cord care, 
initiation of breastfeeding, immunization, eye care, recognition of danger signs, care of the preterm/ low birth weight infant and management of newborn illnesses. Essential newborn care (ENC) is designed to improve health of newborns through a minimum set of interaction that should be made available for all births. 6,7 Essential newborn care is based on simple principles of prevention of infection, thermal protection, resuscitation of newborn with asphyxia, early and exclusive breast feeding, care of low birthweight babies and identification and appropriate referral of sick neonates. ${ }^{(8,9)}$ The care health professionals provide at time of birth is critical in helping to prevent complications and ensuring survival. ${ }^{10}$

Nursing is the profession in the health service that mostly dependent on communication skills. They interact with patients when they are most vulnerable and have to perform intimate procedures. ENC also requires professional skills along with communication related to theoretical and practical knowledge. Being the primary contact personnel, knowledge regarding ENC could have direct impact on neonatal morbidity and mortality. Nurses are the key health workers in newborn healthcare system. Few study about knowledge regarding Essential Newborn Care were done in Bangladesh and neighboring countries. Most of the study was done on mothers knowledge about ENC and very few on health care providers. Training in resuscitation and in essential newborn care reduces perinatal and neonatal mortality. ${ }^{11,12}$ The Helping Babies Breathe and the Essential Care for Every Baby training pro-grams are available, but have not been assessed as needed. The purpose of this study is to assess the knowledge of neonatal care practices among nurses for the development of strategies to improve further. Therefore, essential newborn care should be emphasized in pre-service and in-service nursing and midwifery education. Policy makers thus need to consider these initiatives.

\section{Materials \& Methods:}

This was an observational study conducted at the special Care Neonatal Unit (SCANU) of Chattogram Medical College and two Non-Government Medical
College Hospitals of Chattogram city named Chattogram Maa- O- Shishu Hospital and Bangabandhu Memorial Hospital, University of Science \& Technology Chattogram (USTC) from $1^{\text {st }}$ December 2015 to $31^{\text {st }}$ May 2016. Nurses who were working in the SCANU of the above mentioned study places for at least one month or who had special courses/ training on neonatology were included in the study with their consent. Purposive sampling technique were applied for selecting the sample. Sample size were 100.

A structured data sheet (questionnaire - set on the basis of ENC component) containing 18 multiple choice questions based on knowledge regarding component of ENC was predesigned to collect data. The questionnaire was organized in two parts; sociodemographic characteristics, knowledge of nurses regarding essential newborn care. Knowledge regarding ENC among the nurses graded as satisfactory or not satisfactory. When participant gave right answer it graded as satisfactory if wrong then not satisfactory. Researchers collected data using a structured questionnaire through face-to-face interview.

Data were entered using statistical Package for the Social Sciences (SPSS) version 20.0 for windows. Thereafter data were edited and analyzed. Descriptive statistics computed for demographic variables. ANOVA and Unpaired $t$ test were carried out to find out the association of qualitative and quantitative data of important key variables with adequacy of knowledge as outcome variables respectively. $\mathrm{P}$ value $<0.05$ was considered as significant. For quality assurance professional assistance from a statistician was taken. The study was ethically reviewed and approved by the ethical review committee of Chittagong Medical College Hospital, Chittagong. Institutional clearance was obtained from the Hospital Directors of Chittagong Medical College Hospital and other centers. Singed informed consent was obtained from all participants.

\section{Results:}

This study observed that among 100 nurses more than half $(52.0 \%)$ nurses were belonged to age 21-30 years. Majority $97(97.0 \%)$ were female, 50(50.0\%) were Muslim, $66(66.0 \%)$ were married, $69(69.0 \%)$ had completed 
diploma in nursing and midwife education, 56(56.0\%) worked in non-government hospital and 56(56.0\%) had completed special courses/training in neonatology (Table 3.1). Table 3.2 shows the knowledge of nurses about essential newborn care. Table 3.3 shows mean distribution of knowledge regarding essential newborn care according to nurse's demographic profile. Result shows the knowledge regarding essential newborn care were almost similar with age, gender, religion, marital status, educational level, working place and attending special courses/training in neonatology Table 3.4 shows response, mean and SD score of level of knowledge. $94.75(94.75 \%)$ of the nurses had satisfactory knowledge regarding essential newborn care.

Table-I

Demographic characteristics studied nurses $(n=100)$

Demographic characteristic

Age (years)

$$
\leq 20
$$

21-30

$31-40$

$>40$

Gender

Male

Female

Religion

Islam

Hinduism

Buddhism

Others

Marital status

Married

Unmarried

Educational status

Diploma in nursing

Diploma in nursing and midwife

B. Sc in nursing

Others

Work in

Govt. Hospital

Non Govt. Hospital

Attending special courses/training in neonatology

Yes

No
6.0

52.0

30.0

12.0
3.0

$97 \quad 97.0$

$50 \quad 50.0$

$33 \quad 33.0$

$13 \quad 13.0$

$4 \quad 4.0$

$66 \quad 66.0$

$34 \quad 34.0$

$7 \quad 7.0$

$69 \quad 69.0$

$11 \quad 11.0$

$13 \quad 13.0$

$44 \quad 44.0$

$56 \quad 56.0$

$56 \quad 56.0$

$44 \quad 44.0$ 


\section{Table-II}

Knowledge of nurses about essential newborn care $(n=100)$

Variables

What do you mean by essential newborn care
Immediate proper newborn care

Nurse can't do
Frequency

Percentage

$98 \quad 98.0$

2

Delivery room should be

Well lighted, well ventilated and clean

Any where

98

2

98.0

What type of aseptic techniques you should maintain before handling the baby

Wash with soap water

Wash with other disinfectant solution

42

58

42.0

58.0

When should a baby start spontaneous breathing

Immediately after birth

Few minutes after birth

98

2

98.0

2.0

If baby is well (cry and breath spontaneously), then what you do Put the baby with his/her mother Put baby in the incubator

What should you do it a baby does not breathe spontaneously

Resuscitate the baby

Slapping the back

Hanging upside down

92

92
5

3

92.0

5.0

3.0

How resuscitation can be done

Mouth to mouth breathing and flickering on sole

Suction, Rubbing over the back, use bag and mask ventilation

20

80

20.0

80

80.0

100

100.0

0

0.0

Bath the baby

Care of umbilical cord after delivery

Use Chlorhexidine (7.1\%) for once and keep it Dry

Daily clean the umbilical cord by antiseptic wash

Give antibiotic ointment and bandage over it

90

Within $1^{\text {st }}$ hour of delivery

More than 6 hour

Any advise if breast milk not come

Give formula milk

Pre- lacteal feed (honey, water)

Continue with breast feeding even when milk is not coming

\begin{tabular}{cc}
1 & 1.0 \\
1 & 1.0 \\
98 & 98.0 \\
& \\
92 & 92.0 \\
8 & 8.0 \\
91 & 91.0 \\
9 & 9.0 \\
& \\
18 & 18.0 \\
82 & 82.0 \\
\hline
\end{tabular}

(table continued) 
How can you identify sick baby

Baby is unable to take breast feed, lethargic Respiratory distress,

98 convulsion, deep jaundice, and red foul smelling umbilicus

Does not know

LBW baby means birth weight less than 2500 grams, is it -

Right

Wrong

How stabilizing the temperature of LBW baby

Bath baby in water of appropriate temperature

Put on cloths and cover head and put the baby to mother, skin to skin care ( kangaroo mother care)

Room temperature of 28-30 degrees Celsius

How will you give extra care to low birthweight baby

Skin to skin contact (Kangaroo mother care)

Breast feed early \& frequently

Care for infection prevention

All the above (Skin to skin contact, breast feed early \& frequently and care for infection prevention)

\section{Table-III}

Distribution of nurses knowledge regarding essential newborn care according to number of adequate response to questionnaire by their demographic profile $(n=100)$

\begin{tabular}{|c|c|c|c|c|c|}
\hline \multirow[t]{2}{*}{ Age (in years) } & \multirow[t]{2}{*}{$\mathrm{N}$} & \multicolumn{3}{|c|}{ Adequate response } & \multirow[t]{2}{*}{ P value } \\
\hline & & Mean \pm SD & $\min$ & Max & \\
\hline$\leq 20$ & 6 & $15.83 \pm 2.14$ & 13 & 18 & $0.761^{\mathrm{ns}}$ \\
\hline $21-30$ & 52 & $15.85 \pm 1.51$ & 12 & 18 & \\
\hline $31-40$ & 30 & $16.2 \pm 1.33$ & 14 & 18 & \\
\hline$>40$ & 12 & $15.92 \pm 1.08$ & 14 & 17 & \\
\hline \multicolumn{6}{|l|}{ Gender } \\
\hline Male & 3 & $15.67 \pm 2.52$ & 13 & 18 & $0.675^{\mathrm{ns}}$ \\
\hline Female & 97 & $16.03 \pm 1.43$ & 12 & 18 & \\
\hline \multicolumn{6}{|l|}{ Religion } \\
\hline Islam & 50 & $15.82 \pm 1.71$ & 12 & 18 & \\
\hline Hinduism & 33 & $16.24 \pm 1.23$ & 14 & 18 & $0.568^{\mathrm{ns}}$ \\
\hline Buddhism & 13 & $16.23 \pm 1.01$ & 14 & 18 & \\
\hline Others & 4 & $16.0 \pm 0.82$ & 15 & 17 & \\
\hline \multicolumn{6}{|l|}{ Marital status } \\
\hline Married & 66 & $16.03 \pm 1.38$ & 13 & 18 & $0.922^{\mathrm{ns}}$ \\
\hline Unmarried & 34 & $16.0 \pm 1.61$ & 12 & 18 & \\
\hline \multicolumn{6}{|l|}{ Educational status } \\
\hline Diploma in nursing & 7 & $16.14 \pm 1.07$ & 14 & 17 & \\
\hline Diploma in nursing and midwife & 69 & $16.13 \pm 1.35$ & 13 & 18 & $0.435^{\mathrm{ns}}$ \\
\hline B. Sc in nursing & 11 & $15.3 \pm 1.91$ & 12 & 18 & \\
\hline Others & 13 & $15.9 \pm 1.75$ & 13 & 18 & \\
\hline \multicolumn{6}{|l|}{ Working place } \\
\hline Govt. Hospital & 44 & $15.82 \pm 1.39$ & 12 & 18 & $0.140^{\mathrm{ns}}$ \\
\hline Non Govt. Hospital & 56 & $16.24 \pm 1.41$ & 13 & 18 & \\
\hline \multicolumn{6}{|c|}{ Attending special courses/training in neonatology } \\
\hline Yes & 56 & $16.16 \pm 1.5$ & 12 & 18 & $0.311^{\mathrm{ns}}$ \\
\hline No & 44 & $15.86 \pm 1.41$ & 13 & 18 & \\
\hline
\end{tabular}


Table-IV

Distribution of Level of Knowledge among nurses about Essential Newborn Care ( $n=100)$

\begin{tabular}{lcccc} 
Variables & $\begin{array}{c}\text { Response } \\
\text { Satis-factory }\end{array}$ & $\begin{array}{c}\text { Mean } \\
\text { Not Satis-factory }\end{array}$ & & \\
\hline Meaning of Essential Newborn Care & 98 & 2 & 95.25 & 2.54 \\
Delivery room environment & 98 & 2 & 95.25 & 2.54 \\
Asepsis before handling the baby & 100 & 0 & 98.22 & 1.75 \\
Onset of spontaneous breathing & 98 & 2 & 95.25 & 2.54 \\
About resuscitation & 80 & 20 & 78.3 & 1.68 \\
Measure for warming the baby & 100 & 0 & 95.25 & 2.54 \\
Umbilical cord care & 90 & 10 & 88.2 & 1.66 \\
Initiation of breast feeding & 99 & 1 & 98 & 1 \\
Advice if breast milk not come & 98 & 2 & 95.25 & 2.54 \\
Duration of exclusive breast feeding & 92 & 8 & 91.14 & 0.83 \\
Eye care & 91 & 9 & 89.63 & 1.41 \\
Time of Vaccination & 100 & 0 & 98.22 & 1.75 \\
Identification of sick baby & 98 & 2 & 95.25 & 2.54 \\
Low birthweight baby & 99 & 1 & 98 & 1 \\
Stabilizing temperature of low birthweight baby & 91 & 9 & 89.63 & 1.41 \\
Extra care to low birthweight baby & 84 & 16 & 82.45 & 1.42 \\
\hline
\end{tabular}

\section{Discussion:}

Knowledge is one of the crucial aspects of health systems for ENC practices and lack of knowledge may impede provision of ENC. In this study it was observed that more than half (52.0\%) nurses were belonged to age 21-30 years. Sayed et al. ${ }^{13}$ observed that, nearly two third $(60 \%)$ of the nurses were 30 to less than 40 years old, with mean age were $31 \pm 6$ years. In this present study it was observed that majority $(97.0 \%)$ nurses were female, $50.0 \%$ nurses were Muslim, and $66.0 \%$ nurses were married. Sayed et al. ${ }^{13}$ observed that most of the nurses $(85.7 \%)$ were married. In our country Ayiasi ${ }^{14}$ found that Muslims made up 92.0 percent of the sample.

In this study it was observed that nearly two third (69.0\%) nurses had completed diploma in nursing and midwife education, B. Sc in nursing $11.0 \%$, diploma in nursing $7.0 \%, 13.0 \%$ others, $56.0 \%$ nurses were attending special courses/training in neonatology and $56.0 \%$ nurses were work in non government hospital. Sayed et al. ${ }^{13}$ found the education level $67.1 \%$ of nurses are secondary nursing school graduates while $24.3 \%$ of them have completed their university nursing education and only $8.6 \%$ of them have a technical nursing institute certification. Shinde et al. ${ }^{15}$ found the professional education of study subjects were $45(90 \%)$, Graduate nursing and Midwifery (GNM), 02 (04\%) were B.Sc. (NSG) and $03(06 \%)$ were post B.Sc. (NSG).

In this study, 58(58.0\%) washed their hands with disinfectant solution before handling the baby, $99(99.0 \%)$ mentioned that the baby must be breast feeding within $1^{\text {st }}$ hour of delivery, 92(92.0\%) mentioned that baby must be fed 6 months of exclusive breast feed, 91(91.0\%) said about stabilizing the temperature of LBW baby put on cloths, cover head and put the baby to mother. On the other hand a study conducted in Ethiopia where 13.6\% washed their hand before all deliveries they conducted but $48.9 \%$ did not washed at all ,86.0\% of participants were aware the time of breast feeding initiation, $77.2 \%$ knew duration exclusive breast feeding and greater than $62.1 \%$ kept the baby skin to skin contact with the mother. ${ }^{16}$ In this study all the nurses $(100.0 \%)$ mentioned that the baby should be cleaned and warmed with dry cloth. Other study in India revealed that, $89 \%$ of the providers demonstrated wiping of newborn with dry cloth, $63 \%$ showed putting neonate on warm surface. ${ }^{17}$ Louis et al. ${ }^{18}$ observed that the initial steps of resuscitation, which comprise of drying the baby, suctioning and then stimulating, were also evaluated. 
All the 3 steps were known to only 9 (16\%), while 41 (71\%) knew at least 1 of the 3 steps and $8(13 \%)$ did not know any of these initial steps. Among initial steps, 15 (26\%) did not know how to stimulate the baby if he/she does not cry. All had the practice of tying the cord immediately after delivery. All the 5 C.s required for delivery that means clean hands, clean delivery surface, clean blade, clean cord and clean cord tie were known to only $33(57 \%)$, while $21(36 \%)$ did not know any of them. All of them did hand washing prior to conducting the delivery and used sterile scissors for cutting the cord. Kajale et al. ${ }^{19}$ observed that all the nurses known about early initiation of breast feeding and burping, $80 \%$ knew about breast feeding, $64 \%$ about KMC, $60 \%$ about prevention of sepsis and danger signs. In this study, the mean knowledge regarding essential newborn care was almost alike in difference age group, no significant $(p>0.05)$ association observed with different age group. Similarly, the mean accurate knowledge regarding essential newborn care was not significantly $(p>0.05$ ) associated with gender and religion in this study. Shinde ${ }^{15}$ study found that majority $(60.0 \%)$ of study subject's staff nurses had medium knowledge and 40.0\% of them had adequate knowledge regarding newborn care. The mean accurate knowledge regarding essential newborn care was not significantly $(\mathrm{p}>0.05)$ associated with attending special courses/training in neonatology in this study. Shinde ${ }^{15}$ found that most of study subjects staff nurse had medium knowledge. Regarding the association between attending special courses/training in neonatology with knowledge about essential newborn care, the average correct knowledge was $16.16 \pm 1.5$ in attending special courses/training in neonatology and $15.86 \pm 1.41$ in without attending special courses/training in neonatology. Louis et al. ${ }^{18}$ found that majority of healthcare personnel had good awareness about breastfeeding and clean practices while conducting delivery. In contrast, knowledge about neonatal resuscitation and some aspects of essential newborn care was poor. Sayed et al. ${ }^{13}$ mentioned that education and training are potential means for implementing effective nursing care at Neonatal Intensive Care Unit (NICU), as they alter perception, increase knowledge, and in turn change work practice. Their study revealed that about two third of nurses' knowledge was incompetent about neonates in intensive care unit.

\section{Conclusion}

The study reflects the basic knowledge of nurses working in the SCANU of different hospitals of the second largest city of Bangladesh. Most of the nurses know their job. Though the results appear satisfactory there are lot of areas of improvement and hospital authorities and policy makers should work on it.

\section{Acknowledgements}

I acknowledge my heartiest gratitude to the professor, associate professor assistant professors, doctors, friends and colleagues in the department of Child Health, Chattogram Medical College Hospital for their cooperation and valuable suggestions.

Conflicts of interest: There is nothing to declare

\section{References:}

1. Neonatal mortality 2020. Available from: URL:http:// www.unicef.org

2. Ehret DY, Patterson JK, Bose CL. Improving neonatal care: a global perspective. Clin Perinatol 2017; 44:56782.https://doi.org/10.1016/j.clp.2017.05. 002. PMid: 28802340

3. National Institute of Population Research \& Training (NIPORT), and ICF. Bangladseh Demographic \& Health Survey 2017-18. Dhaka, Bangladesh, and Rockville, Maryland, USA; 2019.

4. The Million Death Study Collaborators Causes of neonatal and child mortality in India [editorial]. Nationally representative mortality survey. Lancet 2010 ; 376:185360. https://doi.org/10.1016/S0140-6736(10)61461-4

5. Ghana Statistical Service (Ghana), Ghana Health Service (GHS) and ICF Macro, Ghana Demographic and Health Survey (GDHS);2008.

6. Essential newborn care-Report of a technical Working Group. WHO /FRH/MSM/96.13. Switzerland Geneva: World Health Organization;1996.

7. Training modules on essential newborn care and breast feeding. Copenhagen, Denmark (Europe): World Health Organization;2002.

8. Castello A, Maenandhar D. Improving newborn health in developing countries. London:Imperial College Press;1998.

9. Strategic directions to improve newborn health in the South-East Asia Region. New Delhi, India: World Health Organization SEAR; 2004.

10. Paul VAD. Newborn nursing for facility based care. Learn Guide 2014.

11. Carlo WA, Goudar SS, Jehan I, Chomba E, Tshefu A, Garces A et al. Newborn-care training and perinatal mortality in developing countries. N Engl J Med 2010; 362:614-23. https://doi.org/10.1056/NEJMsa0806033.PMid:20164485, PMCid:PMC3565382 
12. Carlo WA, McClure EM, Chomba E, Chakraborty H, Hartwell T, Harris $\mathrm{H}$ et al. Newborn care training of midwives and neonatal and perinatal mortality rates in a developing country. Pediatrics 2010;126:1064-71.https:/ /doi.org/10.1542/peds.2009-3464.PMid:20937659

13. Sayed SME, Sabry YY, Sharkawy HM, Elsayed EM, Ali T. Establishing Basic Standards of Nursing care protocol at Neonatal Intensive care unit. Nature and Science 2013;11(4):86-92.

14. Ayiasi RM, Kasasa S, Criel B, Orach CG, Kolsteren P. Is antenatal care preparing mothers to care for their newborns? A community-based cross-sectional study among lactating women in Masindi, Uganda. BMC pregnancy and childbirth 2014;14(1):1. https://doi.org/10.1186/1471-2393-14114.PMid:24667001, PMCid:PMC3987096

15. Shinde S. Knowledge of Staff Nurses in Immediate Care of Newborn Baby and their Implications. A Descriptive Survey Based Study. Bulletin of Pharmaceutical Research 2015;5i(3):108-11.
16. Bayisa BN, Fikadu BH, Asrat DM. Knowledge and Practice of Essential Newborn Care and Associated Factors among Nurses and Midwives Working at Health Centers in Jimma Zone, Ethiopia. Journal of Nursing and Care 2018;7(1).

17. Van WV, Epoo B, Nastapoka J, Harney E. Reclaiming birth, health and community: Midwifery in the Inuit villages of Nunavik, Canada. J Midwifery Womens health 2007;52: 384-91. https://doi.org/10.1016/j.jmwh.2007. 03.025. PMid:17603961

18. Louis D, Kumar P, Gupta A. Knowledge and practices of healthcare providers about essential newborn care and resuscitation in a district of Haryana. J Indian Med Assoc 2013;111(2):114-7.

19. Kazale P, Patil N, Mhaske S. To assess the knowledge about essential newborn care in the nursing staff catering to neonatal population in tertiary care hospital. Vims health science journal 2014;1(2):10-11. 